\title{
Arterial Spin-Labeling Perfusion MR Imaging Demonstrates Regional CBF Decrease in Idiopathic Normal Pressure Hydrocephalus
}

\author{
(D). Virhammar, (D). Laurell, (D)A. Ahlgren, and (DE.-M. Larsson
}

\begin{abstract}
BACKGROUND AND PURPOSE: Regional cerebral blood flow has previously been studied in patients with idiopathic normal pressure hydrocephalus with imaging methods that require an intravenous contrast agent or expose the patient to ionizing radiation. The purpose of this study was to assess regional CBF in patients with idiopathic normal pressure hydrocephalus compared with healthy controls using the noninvasive quantitative arterial spin-labeling MR imaging technique. A secondary aim was to compare the correlation between symptom severity and CBF.
\end{abstract}

MATERIALS AND METHODS: Differences in regional cerebral perfusion between patients with idiopathic normal pressure hydrocephalus and healthy controls were investigated with pseudocontinuous arterial spin-labeling perfusion MR imaging. Twenty-one consecutive patients with idiopathic normal pressure hydrocephalus and 21 age- and sex-matched randomly selected healthy controls from the population registry were prospectively included. The controls did not differ from patients with respect to selected vascular risk factors. Twelve different anatomic ROls were manually drawn on coregistered FLAIR images. The Holm-Bonferroni correction was applied to statistical analyses.

RESULTS: In patients with idiopathic normal pressure hydrocephalus, perfusion was reduced in the periventricular white matter $(P<.001)$, lentiform nucleus $(P<.001)$, and thalamus $(P<.001)$ compared with controls. Cognitive function in patients correlated with $C B F$ in the periventricular white matter $(r=0.60, P<.01)$, cerebellum $(r=0.63, P<.01)$, and pons $(r=0.71, P<.001)$.

CONCLUSIONS: Using pseudocontinuous arterial spin-labeling, we could confirm findings of a reduced perfusion in the periventricular white matter, basal ganglia, and thalamus in patients with idiopathic normal pressure hydrocephalus previously observed with other imaging techniques.

ABBREVIATIONS: $\mathrm{ASL}=$ arterial spin-labeling; $\mathrm{DWMH}=$ deep white matter hyperintensities; $\mathrm{NPH}=$ idiopathic normal pressure hydrocephalus; $\mathrm{MMSE}=$ Mini-Mental State Examination; QRAPMASTER = Quantification of Relaxation Times and Proton density by Multiecho acquisition of a saturation recovery with Turbo spin-echo Readout

diopathic normal pressure hydrocephalus (iNPH) is a condition with balance and gait disturbances, cognitive dysfunction, and urinary incontinence. ${ }^{1}$ The symptoms can be reversed with

Received March 16, 2017; accepted after revision June 12

From the Departments of Neuroscience, Neurology (J.V.) and Surgical Sciences, Radiology (E.-M.L.), Uppsala University, Uppsala, Sweden; Department of Pharmacology and Clinical Neuroscience (K.L.), Unit of Neurology, Östersund, Umeå University, Umeå, Sweden; and Department of Medical Radiation Physics (A.A.), Lund University, Lund, Sweden.

Johan Virhammar was supported by the independent Swedish foundation Erik, Karin och Gösta Selanders Stiftelse; Katarina Laurell received research grants from the Region Jämtland Härjedalen.

Please address correspondence to J. Virhammar, MD, PhD, Department of Neuroscience, Neurology, Uppsala University, Akademiska sjukhuset, ing 85, 751 85 Uppsala, Sweden; e-mail: johan.virhammar@neuro.uu.se; @johanvirhammar

三 Indicates article with supplemental on-line tables and appendix.

Indicates article with supplemental on-line photo.

http://dx.doi.org/10.3174/ajnr.A5347 implantation of a shunt system, with improvement in 50\%-80\% of patients. ${ }^{2-4}$

Regional CBF has been assessed in patients with iNPH to find explanations for the pathophysiology and in search of better methods to identify shunt responders. Several studies have shown reduced perfusion in the frontal cortex, periventricular WM, basal ganglia, thalamus, and cerebellum in patients with iNPH compared with controls, but the results have been divergent. ${ }^{5-11}$ In a recent study, reduced hippocampal CBF was reported as well. ${ }^{9}$

These previous studies have used different methods to estimate CBF: SPECT, PET, DSC MR imaging, and CT perfusion. ${ }^{8,9,12,13}$ These methods require administration of a radioactive tracer or a contrast agent, and SPECT, PET, and CT expose the patient to ionizing radiation.

Pseudocontinuous arterial spin-labeling (ASL) is an MR imaging technique with the advantage of allowing quantitative mea- 


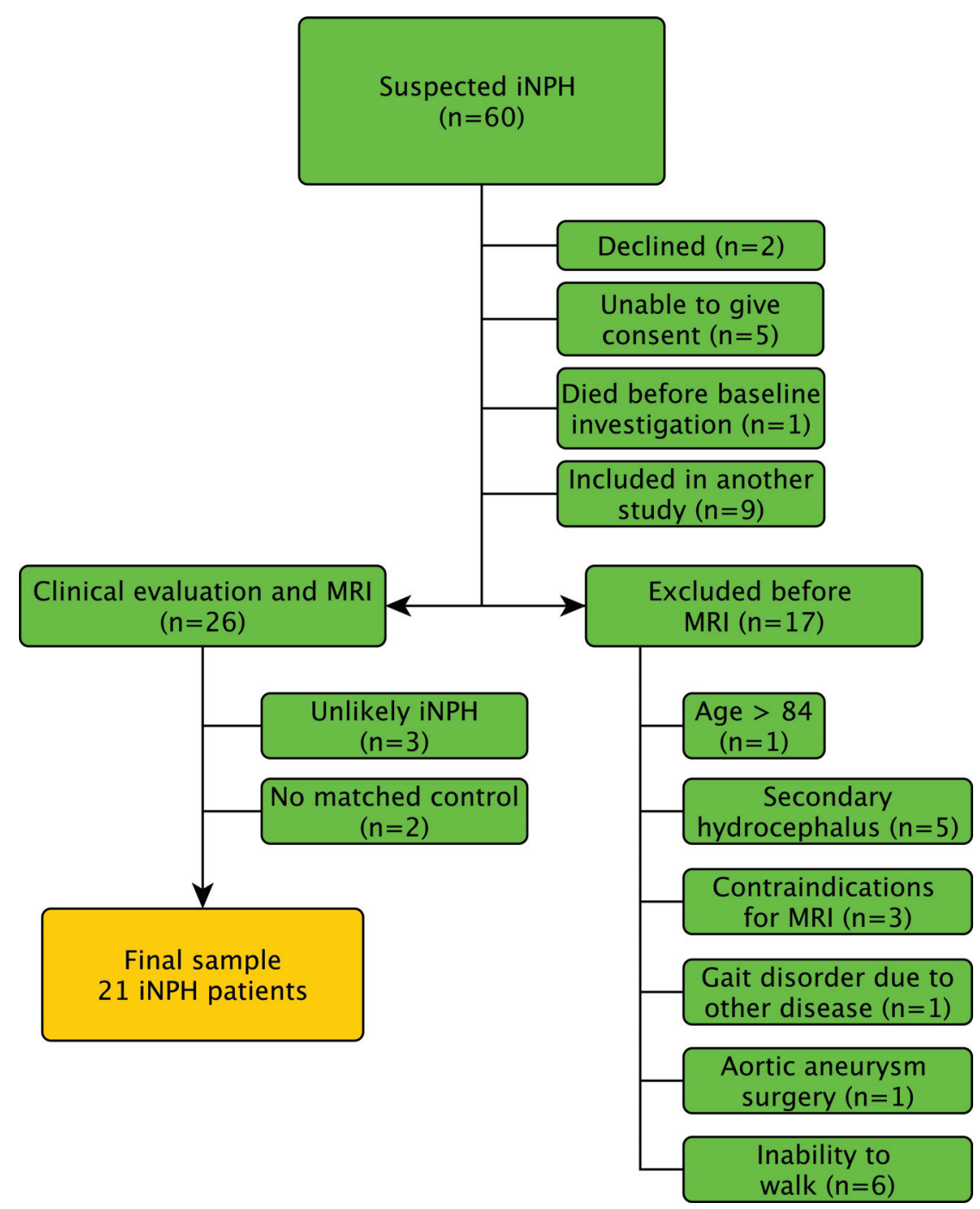

FIG 1. Flow chart describing the inclusion process of patients.

surements of CBF without injection of any contrast agent. ${ }^{14}$ The method is completely noninvasive, with a relatively short scan time ( $\sim 5$ minutes) and can therefore easily be added to a standard MR imaging protocol. To the best of our knowledge, no previous study has investigated differences in CBF between healthy controls and patients with iNPH with an ASL technique.

The purpose of this study was to use ASL to investigate whether regional cerebral perfusion is reduced in patients with iNPH compared with healthy controls and whether the level of clinical symptoms correlates with $\mathrm{CBF}$.

\section{MATERIALS AND METHODS \\ Patients and Healthy Controls}

Sixty patients were prospectively and consecutively recruited from the waiting list of patients referred to Uppsala University Hospital for evaluation of suspected iNPH. Nine patients were excluded for participating in an ongoing multicenter study of the CSF tap test. Patients with complete inability to walk were excluded because of the intention to study the relationship between $\mathrm{CBF}$ and gait and balance function. Other exclusion criteria were secondary or congenital hydrocephalus, contraindications to MR imaging, older than 84 years of age, severe cognitive dysfunction with an inability to give informed consent, refusal to undergo evaluation or shunt surgery, and gait problems that could be explained by other known diseases. The inclusion process is illustrated in the flow chart (Fig 1). Of the 26 patients examined clinically, 17 were classified as having probable iNPH according to the iNPH guidelines. ${ }^{15}$ Six patients were classified as having possible iNPH, 1 because of only 1 triad symptom (gait disturbance), 1 due to severe white matter changes, 1 because of a small cyst communicating with 1 of the lateral ventricles, and 3 due to a history of head trauma, probably unrelated to the hydrocephalus. Three patients were considered unlikely to have iNPH, 2 because of symptoms of Parkinson disease and 1 with an aqueductal stenosis. The patients examined in this study have been described in detail in a previous study. ${ }^{16}$

Controls were randomly recruited from the county of Uppsala using the Swedish population registry and were matched with patients with respect to age ( \pm 2 years) and sex. Exclusion criteria were any known neurologic disease, stroke, diabetes mellitus, history of myocardial infarction that required acute treatment or resulted in persistent electrocardiogram changes, dependence on walking aids, or any terminal disease. Antihypertensive medication, aspirin, and common pain medications were allowed. Information was sent to 105 potential elderly controls. Forty-nine did not reply; 31 responded that they did not want to participate or could not participate according to the exclusion criteria. The remaining 25 accepted participation and were examined clinically by a neurologist. MR imaging examination was not possible in 2 of the 25 controls because the MR imaging scanner was upgraded before those 2 controls were examined, and another 2 controls did not match any patient. Thus, a total of 21 matched pairs of patients ( 15 with probable iNPH and 6 with possible iNPH) and controls were available and included in the statistical analysis. The study was approved by the local ethics committee in Uppsala, Sweden.

\section{Clinical Examination}

Neurologists, especially trained physiotherapists, and occupational therapists performed the clinical examinations. Besides a standard neurologic examination, the tests included the modified Rankin Scale, Mini-Mental State Examination (MMSE), incontinence scale, ${ }^{17}$ Romberg test, and time and number of steps required to walk $10 \mathrm{~m}$ at a maximum pace. Tests of motor function were performed twice, and the mean result was used in the statistical analyses. 


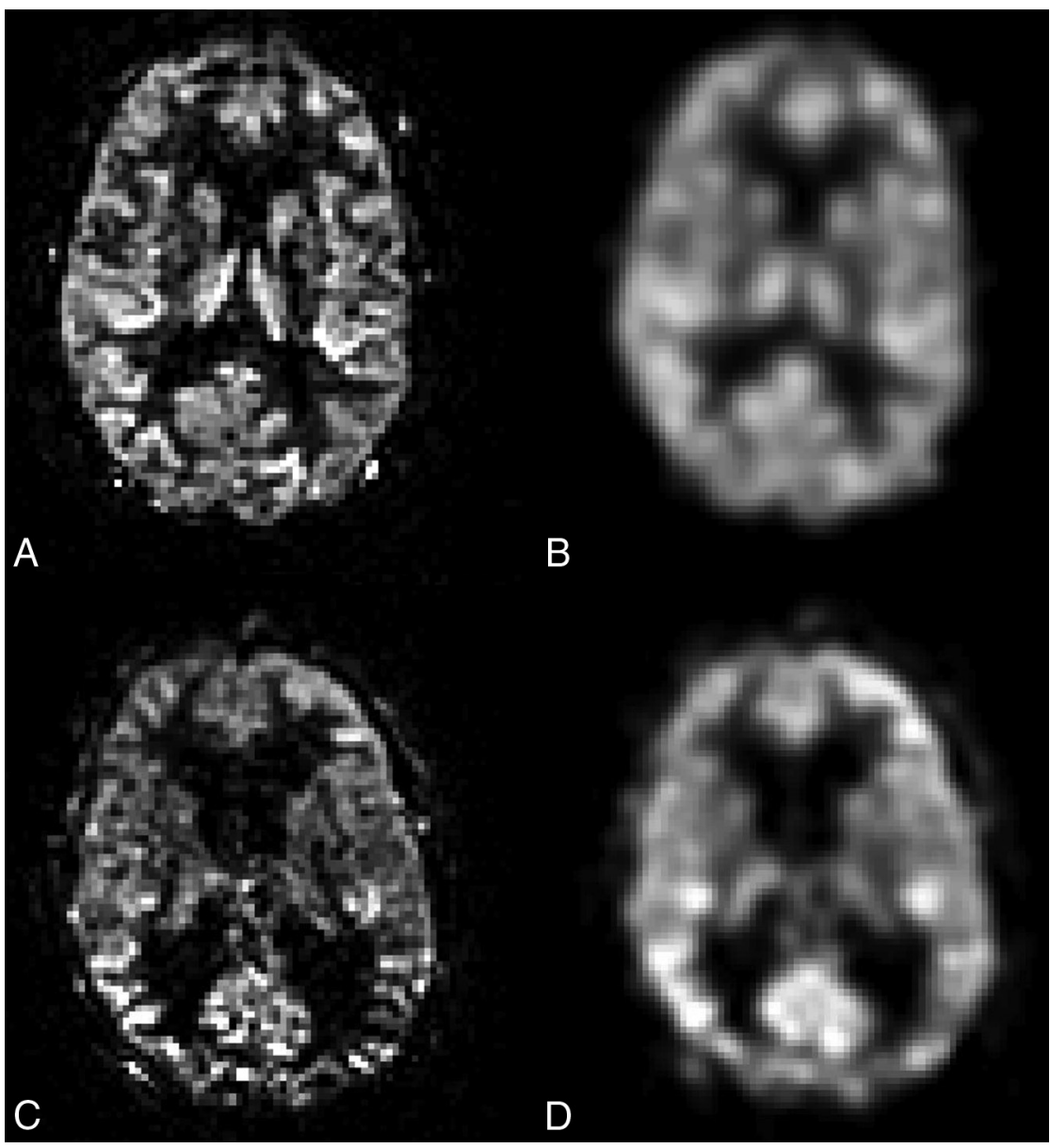

FIG 2. Example of ASL perfusion maps from a healthy control without $(A)$ and with $(B)$ smoothing, as well as from a patient with iNPH without $(C)$ and with $(D)$ smoothing.

\section{Imaging}

Details of the imaging method, scanner protocol, and postprocessing have been described previously. ${ }^{16}$ The first MR imaging was performed between 8:00 AM and 10:00 AM. The second MR imaging was performed 60 minutes after the first scan to assess the repeatability of the method. The patients left the scanner after the first investigation. Between the investigations, the patients rested in the supine position in a quiet, isolated room next to the scanner. The patients were not allowed to use caffeine on the day of the MR imaging, and all transportation was performed with the patient in bed or in a wheelchair to minimize variations in CBF. Healthy controls were only examined once with MR imaging.

\section{Scanner and Imaging Protocol}

Imaging was performed with a 3T MR imaging scanner (Achieva; Philips Healthcare, Best, the Netherlands) with a 32-channel head coil. A 3D T1-weighted gradient-echo and a T2-weighted FLAIR sequence were included in the protocol. Perfusion MR imaging was performed with a background-suppressed pseudocontinuous ASL sequence, with a single-shot echo planar imaging readout, with the following protocol: $\mathrm{FOV}=264 \times 264 \mathrm{~mm}^{2}$, in-plane resolution $=2.75 \times 2.75 \mathrm{~mm}^{2}$, section thickness $=5 \mathrm{~mm}$, number of sections $=20, \mathrm{TR}=4100 \mathrm{~ms}, \mathrm{TE}=14.5 \mathrm{~ms}$, label duration $=1650 \mathrm{~ms}$, postlabeling delay $=1600 \mathrm{~ms}$, and 30 repetitions.
To allow quantitative $\mathrm{CBF}$ values, we obtained a fast reference scan.

Synthetic MR imaging data were acquired with the multisection, multiecho, and multisaturation delay sequence Quantification of Relaxation Times and Proton density by Multiecho acquisition of a saturation recovery with Turbo spinecho Readout (QRAPMASTER). ${ }^{18} \mathrm{Be}-$ cause of technical problems, synthetic MR imaging data were not acquired in 2 patients and in 1 control.

\section{Perfusion MR Imaging Postprocessing}

For perfusion quantification, the compartment model proposed by Alsop and Detre was used. ${ }^{19}$ The magnetization difference (perfusion-weighted signal) is given by

$$
\Delta M=2 M_{0 a} \alpha f T_{1 a}\left(1-e^{-\tau / T_{1 a}}\right) e^{-w / T_{1 a}},
$$

where $M_{0 a}$ is the arterial blood magnetization, $\alpha$ is the labeling efficiency, $f$ is the perfusion, $T_{1 a}$ is the T1 of arterial blood, $\tau$ is the labeling duration, and $w$ is the postlabeling delay. Labeling efficiency was set to $70 \%, T_{1 a}$ was set to $1664 \mathrm{~ms}$, and $M_{\mathrm{Oa}}$ was estimated with the signal intensity of CSF (see Alsop and Detre ${ }^{19}$ for more detail).

As an anatomic reference, the FLAIR images were coregistered to the perfusion maps by using a 12-degree affine transform (elastix; http://elastix.isi.uu. $\mathrm{nl} /$ ). To minimize the effect of nonideal image coregistration and to reduce the intrasubject variation caused by varying delineation of regions, we spatially smoothed the CBF maps (Gaussian kernel with 4-mm full width at half maximum, Fig 2).

\section{ROIs and Morphologic Evaluations}

Twelve different ROIs were drawn manually on transverse T2 FLAIR images using the in-house software Eval Gui (developed by Markus Nilsson, Lund University, Lund, Sweden). We covered the following regions: cerebellum, pons, high-convexity cortex, medial frontal cortex, lentiform nucleus, medial temporal lobe, supplementary motor area, thalamus, frontal WM, lateral WM, superior WM, and periventricular WM. The number of sections and voxels for all ROIs is listed in Table 1. Four different ROIs are illustrated in Fig 3, and all ROIs in both patients and controls are illustrated in the On-line Appendix. All sections of all scans were visually inspected, and care was taken not to include artifacts or large vessels in the ROIs. The ROIs were drawn by the first author (J.V., 4 years of experience) and reviewed by the last author (E.-M.L., $>30$ years of experience). When ROIs were drawn, the investigator was blinded to the patients' clinical data. CBF values were calculated for every voxel, and a mean $\mathrm{CBF}$ was provided in each ROI.

For descriptive purposes, we assessed the following morphologic 
Table 1: Cerebral blood flow values in patients and controls ${ }^{a}$

\begin{tabular}{|c|c|c|c|c|c|c|}
\hline \multirow[b]{2}{*}{ ROI } & \multirow[b]{2}{*}{ Sections } & \multirow[b]{2}{*}{ Voxels ${ }^{b}$} & \multirow[b]{2}{*}{ CBF, Repeatability ${ }^{C}$} & \multicolumn{2}{|c|}{$\begin{array}{l}\text { CBF (Mean) (SD) } \\
\text { (mL/100 g/min) }\end{array}$} & \multirow[b]{2}{*}{$P$ Value $^{\mathrm{d}}$} \\
\hline & & & & Patients & Controls & \\
\hline Cerebellum & 2 & 924 (144) & 0.92 & $20.7(9.3)$ & $27.2(7.0)$ & .006 \\
\hline MFC & 3 & $133(30)$ & 0.90 & $24.9(5.6)$ & $27.4(5.1)$ & .23 \\
\hline Lentiform nucleus & 1 & 115 (15) & 0.75 & $20.4(2.7)$ & $25.0(4.0)$ & $<.001^{\mathrm{e}}$ \\
\hline MTL & 1 & 114 (15) & 0.86 & $30.4(5.6)$ & $31.7(3.8)$ & .86 \\
\hline $\mathrm{HCC}$ & 2 & 1350 (459) & 0.72 & $22.5(5.5)$ & $21.6(3.0)$ & .47 \\
\hline Pons & 3 & 68 (17) & 0.90 & $22.0(7.0)$ & $24.1(5.3)$ & .19 \\
\hline SMA & 2 & $130(18)$ & 0.72 & $25.1(5.1)$ & $25.2(3.7)$ & .93 \\
\hline Thalamus & 2 & $116(19)$ & 0.92 & $26.8(10.2)$ & $34.1(5.6)$ & $<.001^{\mathrm{e}}$ \\
\hline Frontal WM & 2 & $41(8)$ & 0.91 & $11.0(5.0)$ & $10.7(2.2)$ & .76 \\
\hline Lateral WM & 2 & $136(33)$ & 0.89 & $12.2(3.0)$ & $13.8(1.7)$ & .014 \\
\hline Superior WM & 2 & $67(7)$ & 0.88 & $14.2(5.0)$ & $12.6(2.5)$ & .17 \\
\hline Periventricular WM & 1 & $186(15)$ & 0.88 & $8.0(2.5)$ & $13.1(3.1)$ & $<.001^{\mathrm{e}}$ \\
\hline
\end{tabular}

Note:- HCC indicates high-convexity cortex; MFC, medial frontal cortex; MTL, medial temporal lobe; SMA, supplementary motor area.

${ }^{a}$ Data are mean with SD in parentheses unless otherwise indicated.

${ }^{b}$ Mean (SD) voxels in each ROI in patients.

' Repeatability of perfusion measurements between the 2 baseline investigations using the intraclass correlation coefficient.

dUnadjusted $P$ values tested with the paired-samples $t$ test.

e Significant after Holm-Bonferroni correction.

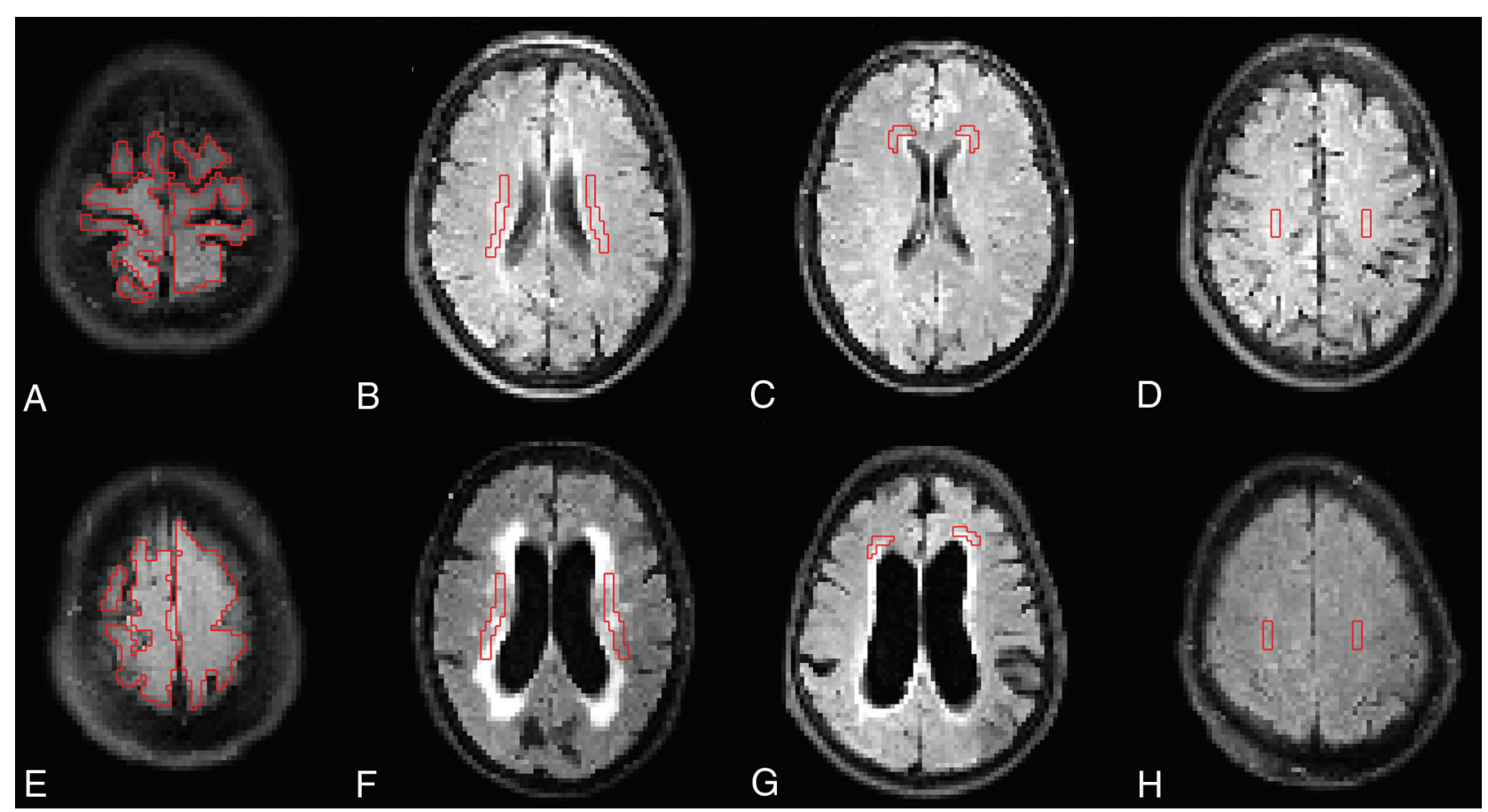

FIG 3. FLAIR images that were coregistered to the $A S L$ images and used to draw ROIs. $A-D$, Healthy controls. $E-H$, Patients with iNPH. $A$ and $E$, High-convexity cortex. B and F, Lateral periventricular WM. C and G, Frontal WM. D and H, Superior WM. All ROls above were drawn in 2 adjacent sections, 1 of which is shown here.

MR imaging features: the Evans index, ${ }^{20}$ the callosal angle, ${ }^{21}$ and deep white matter hyperintensities (DWMH) according to the visual grading scale of Fazekas et al. ${ }^{22}$ Disproportionately enlarged subarachnoid space hydrocephalus was defined as a combination of enlarged ventricles (Evans index of $>0.3$ ), narrow sulci at the high convexity or parafalcine and dilated Sylvian fissures (Table 2). ${ }^{3}$ The measurements of morphologic features were performed by the last author (E.-M.L.). She was blinded to CBF data.

\section{Volumetric Measurement}

Measurements of CSF volumes were performed by using the QRAPMASTER sequence, ${ }^{18}$ which provides quantification of longitudinal relaxation time (T1), transverse relaxation time (T2), and proton density. The software SyMRI Brain Studio (SyntheticMR, Linköping, Sweden) uses combinations of T1, T2, and proton density to estimate voxelwise partial volumes of GM, WM, and CSF, which allow volumetric estimation of these tissues. The volume of both lateral ventricles was calculated semiautomatically in both patients and controls after manual delineation on each section that included the lateral ventricles.

\section{Statistical Analysis}

The mean CBF in each ROI from the 2 scans of the patients was used in the statistical analyses. Each patient was paired with an 
Table 2: Demographics and background data in patients and controls ${ }^{\mathrm{a}}$

\begin{tabular}{lccc}
\hline & Patients $(\boldsymbol{n}=\mathbf{2 1})$ & Controls $(\boldsymbol{n}=21)$ & $\boldsymbol{P}$ Value \\
\hline Age (median) (range) & $74(65-81)$ & $74(65-82)$ & $\mathrm{NS}^{\mathrm{b}}$ \\
No. of men (\%) & $11(52 \%)$ & $11(52 \%)$ & $\mathrm{NS}^{\mathrm{c}}$ \\
MMSE & $25(22-27)$ & $30(29-30)$ & $<.001^{\mathrm{d}}$ \\
Urgency scale & $3(1-4)$ & $1(1-1)$ & $<.001^{\mathrm{d}}$ \\
Modified Rankin Scale score & $2(2-3)$ & $0(0-0)$ & $<.001^{\mathrm{d}}$ \\
Romberg test (sec) & $18(4-60)$ & $60(60-60)$ & $<.001^{\mathrm{d}}$ \\
10-m Walk time (sec) & $12(8-17)$ & $5(5-6)$ & $<.001^{\mathrm{d}}$ \\
10-m Walk (No. of steps) & $22(16-30)$ & $12(11-13)$ & $<.001^{\mathrm{d}}$ \\
Evans index score & $0.35(0.34-0.39)$ & $0.28(0.24-0.30)$ & $<.001^{\mathrm{d}}$ \\
DWMH & $1(1-3)$ & $1(1-2)$ & $\mathrm{NS}^{\mathrm{d}}$ \\
DESH (No.) (\%) & $14(67 \%)$ & $0(0 \%)$ & $<.001^{\mathrm{c}}$ \\
Callosal angle & $66^{\circ}\left(60^{\circ}-73^{\circ}\right)$ & $113^{\circ}\left(104^{\circ}-121^{\circ}\right)$ & $<.001^{\mathrm{d}}$ \\
Lateral ventricular volume $(\mathrm{mL})$ & $130(111-136)$ & $31(24-54)$ & $<.001^{\mathrm{d}}$ \\
\hline
\end{tabular}

Note:-DESH indicates disproportionately enlarged subarachnoid space hydrocephalus; NS, not significant.

a Unless indicated otherwise, data are median, with interquartile range in parentheses.

${ }^{b}$ Mann-Whitney $U$ test.

${ }^{c} \mathrm{McNemar}$ test.

${ }^{\mathrm{d}}$ Wilcoxon signed rank test.

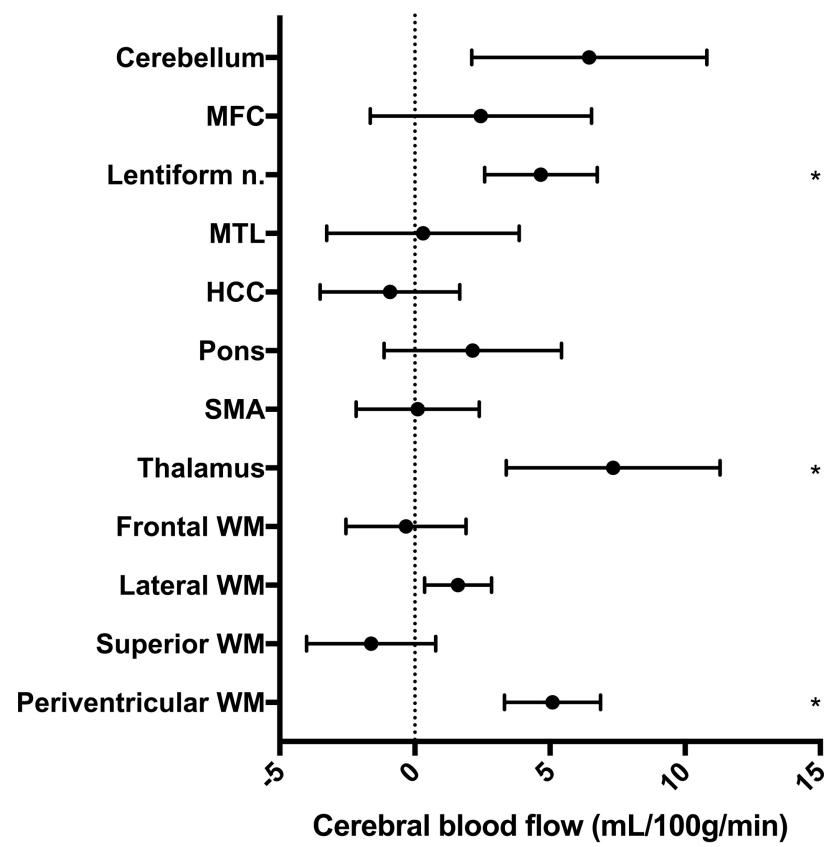

FIG 4. Mean difference with $95 \% \mathrm{Cl}$ between patients with iNPH and healthy controls. MFC indicates the medial frontal cortex; HCC, highconvexity cortex; MTL, medial temporal lobe; SMA, supplementary motor area. The asterisk indicates a significant difference after HolmBonferroni correction.

age- and sex-matched control. A paired-samples $t$ test was used for the difference in CBF between patients and controls. Differences in descriptive data and comorbidity were analyzed with the Wilcoxon signed rank test or the McNemar test. Difference in age was tested with the Mann-Whitney $U$ test. Correlations were assessed with the Spearman correlation. An intraclass correlation coefficient (2-way mixed, single measures) was used to calculate the repeatability of CBF between the 2 investigations in patients. For the analysis of CBF in patients and controls in the 12 ROIs, the Holm-Bonferroni correction for multiple comparisons was applied to reduce type I errors. Holm-Bonferroni correction works by sorting the N $P$ values from the multiple tests and testing the smallest $P$ value against $\alpha / \mathrm{N}$, the second smallest against $\alpha /(\mathrm{N}-1)$, and so on. As soon as a null hypotheses is not rejected, all subse- quent null hypotheses are also deemed not rejected, resulting in an effective significance threshold. With $\alpha=.05$, the effective significance threshold for ROI analysis in patients and controls was $P<$ .006. Correlations were tested among all 12 ROIs, and the 4 clinical tests as well as the 4 morphologic imaging features. Only correlations with $r>0.60$ were considered strong and included in the results section. All analyses were performed with SPSS Statistics for Macintosh, Version 22.0 (IBM, Armonk, New York).

\section{RESULTS \\ Background Data}

The radiologic morphologic measurements and the results of gait and balance tests, urgency, and MMSE differed markedly between patients and healthy controls (Table 2). However, there were no significant differences between patients and controls concerning age, the radiologic finding DWMH, and the use of antihypertensive drugs, acetylsalicylic acid, antidiabetic drugs, or statins, and there were no differences in the history of stroke, traumatic brain injury, meningitis, and previous or present smoking.

\section{Cerebral Blood Flow}

The CBF in all patients and controls is presented in Table 1. In patients, the $\mathrm{CBF}$ was significantly lower in the periventricular WM, lentiform nucleus, and thalamus after Holm-Bonferroni correction (Fig 4). In 3 patients with iNPH, the ASL maps contained vascular artifacts and apparent hypoperfusion in the brain parenchyma. If these 3 patients were excluded from statistical analyses, CBF was still significantly lower in patients in the periventricular WM, lentiform nucleus, and thalamus. There was no difference in age or symptom severity among the 3 patients with vascular artifacts compared with the other patients.

\section{Morphologic Features and CBF}

In controls, there were negative correlations between the Evans index and perfusion in frontal WM $(r=-0.75, P<.001)$, lateral $\mathrm{WM}(r=-0.73, P<.001)$, and periventricular WM $(r=-0.66$, $P<.01)$. In controls, there were also negative correlations between quantified lateral ventricular volume with Synthetic MR imaging and perfusion in the frontal WM $(r=-0.72, P<.001)$, lateral WM $(r=-0.67, P<.01)$, periventricular WM $(r=-0.65$, $P<.01)$, and medial frontal cortex $(r=-0.61, P<.01)$. There were no correlations between ventricular volume and CBF in patients.

\section{Clinical Symptoms and CBF}

In patients, the MMSE score correlated significantly with CBF in the pons $(r=0.71, P<.001)$, cerebellum $(r=0.63, P<.01)$, and periventricular WM $(r=0.60, P<.01)$, but not in controls. Neither in patients nor in controls did gait, balance, or urgency incontinence correlate with CBF values. See On-line Tables 1 and 2 for all correlation coefficients in patients.

AJNR Am J Neuroradiol 38:2081-88 Nov 2017 www.ajnr.org 


\section{Repeatability}

Repeatability for the CBF values obtained in the 2 investigations in patients is presented in Table 1 as intraclass correlation coefficients. The intraclass correlation coefficients were in the range of 0.72-0.92.

\section{Volumetric Measurement}

The median lateral ventricular volume in patients was significantly larger than in controls, $130 \mathrm{~mL}$ (interquartile range $=111-$ $136 \mathrm{~mL}$ ) and $31 \mathrm{~mL}$ (interquartile range, $24-54 \mathrm{~mL}$ ), respectively.

\section{DISCUSSION}

In this study, pseudocontinuous ASL, a noninvasive perfusion method without ionizing radiation or contrast agent, was used to compare cerebral perfusion between patients with iNPH and healthy controls. In agreement with previous studies using anatomic ROIs in patients with iNPH, CBF was reduced in the periventricular WM, basal ganglia, and thalamus. ${ }^{5,9}$ There was a trend indicating that CBF was also reduced in the cerebellum in patients with iNPH, but the difference was not significant after Holm-Bonferroni correction. In patients, MMSE correlated significantly with $\mathrm{CBF}$ in the pons, cerebellum, and periventricular WM. The repeatability of the CBF measurements was acceptable to high, which strengthens our findings.

In the present study, reduced CBF in patients with iNPH was found in the basal ganglia, thalamus, and periventricular WM, whereas previous studies also reported hypoperfusion in the frontal cortex, temporal lobes, and cerebellum. 5,9,13 The more conservative correction for multiple analyses performed in our study could be one explanation for this difference, but another could be differences in the selection of healthy controls as well as the difference in imaging methods.

The mechanism of the reduced regional CBF in iNPH is likely multifactorial. One hypothesis is that transependymal passage of CSF into the parenchyma leads to reversal of interstitial fluid flow, initiating local CSF edema in the periventricular WM. The accumulation of interstitial fluid may cause local compression of small vessels and, more important, reduce elimination of vasoactive metabolites. The region mainly affected by hypoperfusion, according to this hypothesis, should be the periventricular WM, which is in line with our results and has also been reported by others. ${ }^{6,9}$ The reduced CBF in the thalamus and lentiform nucleus may be an indirect result of periventricular WM edema, by affecting the penetrating arteries that supply the thalamus and lentiform nucleus.

The supplementary motor area, basal ganglia, thalamus, mesencephalon, or the white matter tracts connecting these regions have been suggested as the most important anatomic structures behind gait and balance disturbances in patients with iNPH. ${ }^{23,24}$ There were no correlations between gait function and CBF in the present study. These findings indicate that the etiology of the gait disturbance in iNPH is complex and might be caused by factors other than reduced CBF.

However, there were correlations in the present study between impaired cognitive function measured with MMSE and reduced CBF in the pons, cerebellum, and periventricular WM. Perfusion in the brain stem has not been well-studied in iNPH, but Tullberg et $\mathrm{al}^{25}$ reported a relative $\mathrm{CBF}$ increase in the mesencephalon in patients with improved wakefulness after shunt surgery. Disturbances in cognitive function, especially in attention and executive ability, have been reported after insults to the pons, ${ }^{26}$ and such symptoms are often seen in iNPH as well. ${ }^{27}$ The connection between impaired cognitive function and reduced CBF in the cerebellum is harder to explain; however, there are reports that the cerebellum is involved in cognitive function. ${ }^{28,29}$

Hypertension and vascular risk factors ${ }^{30}$ and MR imaging evidence of vascular disease such as DWMH are overrepresented in patients with iNPH. ${ }^{31}$ These vascular risk factors are associated with reduced $\mathrm{CBF}^{32}$; therefore, differences in $\mathrm{CBF}$ could be overestimated if controls are a highly selected sample of healthy elderly. In the present study, the controls were randomly selected from the population, with a limited number of exclusion criteria. There was no significant difference in the burden of DWMH or the use of acetylsalicylic acid or antihypertensive drugs between patients and controls, which strengthens our findings.

In controls, there were correlations between the size of the lateral ventricles and reduced CBF in WM regions. A potential reason may be asymptomatic small-vessel disease with reduction of WM volume and secondary dilation of the lateral ventricles, indicating that even moderately enlarged ventricles may not be considered a normal aging phenomenon.

The pseudocontinuous ASL technique has some advantages compared with other perfusion methods. The repeatability in healthy controls and in patients with Alzheimer disease is high, and CBF values have been validated against PET. ${ }^{33}$ Like PET, perfusion values can be quantified. Therefore, no reference regions are needed to calculate relative perfusion values. In SPECT and DSC perfusion, relative values are used, with the risk that pathologic perfusion in the reference regions may affect the relative perfusion values. In a recent study, CT perfusion was evaluated in iNPH and may be a promising alternative because it is available in many sites and is not as sensitive to artifacts from the shunt valve after shunt implantation as with MR imaging. ${ }^{8}$

In the iNPH guidelines from 2005, the SPECT-acetazolamide challenge is the only perfusion method mentioned as a supportive diagnostic technique. ${ }^{15}$ A SPECT-acetazolamide challenge requires a separate imaging investigation, is time-consuming, and exposes the patient to ionizing radiation from the injected tracer. The ASL sequence is rapid and noninvasive; these features make it ideal for research studies in patients with iNPH, but the diagnostic value of the method is uncertain. Although CBF differences were found between patients and controls on a group level, the effect may not be large enough in individual cases to allow the use of ASL as a supportive diagnostic technique in clinical routine.

The strength of this study was that prospectively and consecutively included patients were compared with age- and sex-matched controls who were randomly selected from the population.

Some limitations need to be considered. Three patients had a high intravascular signal with low signal intensity in the brain parenchyma, probably due to a longer arterial transit time than postlabeling delay. ${ }^{34}$ Statistical analysis with and without these 3 patients did not show any significant differences; therefore, we chose not to exclude them. Individually optimized postlabeling 
delays would be preferable in elderly patients with pathologic CBF.

The CBF values in both our patients and controls were low compared with those reported in PET studies, ${ }^{5,35}$ but they were in line with other ASL studies in patients with iNPH. ${ }^{36}$ Also, it has been reported that quantified perfusion values are underestimated in elderly individuals with ASL. ${ }^{37}$ Our main results were probably not affected by this limitation because we compared patients with age-matched controls.

White matter perfusion estimation with ASL is challenging, due to a longer arterial transit time and lower blood volume compared with gray matter. Thus, the WM perfusion signal had a low signal-to-noise ratio, and the corresponding results should be treated with some caution. On the other hand, 2 recent studies suggest that though pixel-wise analysis is challenging, ROI-based analysis of WM perfusion is feasible in single subjects. ${ }^{38,39}$ This finding increases the credibility of our group-level results based on ROI analysis in 21 patients and 21 controls.

Spatial smoothing was applied to reduce the effects of nonideal coregistration but can potentially introduce partial volume effects on CBF measurements. This might have influenced the results of small ROIs with contamination of data from surrounding tissue.

The MMSE cognitive test used in this study may underestimate subcortical deficits that are very relevant in iNPH. More sophisticated neuropsychological tests could have revealed more information about the relationship between cognition and CBF in both patients and controls. ${ }^{40}$

\section{CONCLUSIONS}

Pseudocontinuous ASL was used to compare perfusion of patients with iNPH versus healthy controls. In patients, perfusion values were reduced in the periventricular WM, basal ganglia, and thalamus, and there was a correlation between cognitive dysfunction and reduced CBF. Because ASL is a noninvasive quantitative perfusion method without ionizing radiation or contrast agent, it is a suitable perfusion method for research studies in patients with iNPH.

\section{ACKNOWLEDGMENTS}

The authors thank Markus Nilsson for the software Eval Gui, Markus Fahlström for technical assistance, and Emma Jansson for assistance with examinations of healthy controls. The authors also thank our normal pressure hydrocephalus team and the MR imaging staff at Uppsala University Hospital, especially Britt-Mari Bolinder. Finally, the authors thank Selanders Stiftelse for its support.

Disclosures: Johan Virhammar-RELATED: Grant: Selanders Foundations, Comments: Swedish independent foundation*; UNRELATED: Payment for Lectures Including Service on Speakers Bureaus: Medtronic, Comments: I have received 1 educational lecture honoraria from Medtronic for 1 lecture. André Ahlgren—UNRELATED: Grant: Swedish Research Council.* *Money paid to the institution.

\section{REFERENCES}

1. Adams RD, Fisher CM, Hakim S, et al. Symptomatic occult hydrocephalus with "normal" cerebrospinal-fluid pressure: a treatable syndrome. N Engl J Med 1965;273:117-26 CrossRef Medline

2. Klinge P, Hellström P, Tans J, et al. One-year outcome in the Euro- pean multicentre study on iNPH. Acta Neurol Scand 2012;126: 145-53 CrossRef Medline

3. Hashimoto M, Ishikawa M, Mori E, et al; Study of INPH on neurological improvement (SINPHONI). Diagnosis of idiopathic normal pressure hydrocephalus is supported by MRI-based scheme: a prospective cohort study. Cerebrospinal Fluid Res 2010;7:18 CrossRef Medline

4. Sundström N, Malm J, Laurell K, et al. Incidence and outcome of surgery for adult hydrocephalus patients in Sweden. Br JNeurosurg 2017;31:21-27 CrossRef Medline

5. Owler BK, Momjian S, Czosnyka Z, et al. Normal pressure hydrocephalus and cerebral blood flow: a PET study of baseline values. J Cereb Blood Flow Metab 2004;24:17-23 CrossRef Medline

6. Momjian S, Owler BK, Czosnyka Z, et al. Pattern of white matter regional cerebral blood flow and autoregulation in normal pressure hydrocephalus. Brain 2004;127:965-72 CrossRef Medline

7. Sasaki H, Ishii K, Kono AK, et al. Cerebral perfusion pattern of idiopathic normal pressure hydrocephalus studied by SPECT and statistical brain mapping. Ann Nucl Med 2007;21:39-45 CrossRef Medline

8. Ziegelitz D, Arvidsson J, Hellström P, et al. Pre-and postoperative cerebral blood flow changes in patients with idiopathic normal pressure hydrocephalus measured by computed tomography (CT) perfusion. J Cereb Blood Flow Metab 2016;36:1755-66 CrossRef Medline

9. Ziegelitz D, Starck G, Kristiansen D, et al. Cerebral perfusion measured by dynamic susceptibility contrast MRI is reduced in patients with idiopathic normal pressure hydrocephalus. J Magn Reson Imaging 2014;39:1533-42 CrossRef Medline

10. Corkill RG, Garnett MR, Blamire AM, et al. Multi-modal MRI in normal pressure hydrocephalus identifies pre-operative haemodynamic and diffusion coefficient changes in normal appearing white matter correlating with surgical outcome. Clin Neurol Neurosurg 2003;105:193-202 CrossRef Medline

11. Owler BK, Pena A, Momjian S, et al. Changes in cerebral blood flow during cerebrospinal fluid pressure manipulation in patients with normal pressure hydrocephalus: a methodological study. J Cereb Blood Flow Metab 2004;24:579-87 CrossRef Medline

12. Owler BK, Pickard JD. Normal pressure hydrocephalus and cerebral blood flow: a review. Acta Neurol Scand 2001;104:325-42 CrossRef Medline

13. Larsson A, Bergh AC, Bilting M, et al. Regional cerebral blood flow in normal pressure hydrocephalus: diagnostic and prognostic aspects. Eur J Nucl Med 1994;21:118-23 Medline

14. Wu WC, Fernández-Seara M, Detre JA, et al. A theoretical and experimental investigation of the tagging efficiency of pseudocontinuous arterial spin labeling. Magn Reson Med 2007;58:1020-27 CrossRef Medline

15. Relkin N, Marmarou A, Klinge P, et al. Diagnosing idiopathic normal-pressure hydrocephalus. Neurosurgery 2005;57:S4-16; discussion ii-v Medline

16. Virhammar J, Laurell K, Ahlgren A, et al. Idiopathic normal pressure hydrocephalus: cerebral perfusion measured with pCASL before and repeatedly after CSF removal. J Cereb Blood Flow Metab 2014;34: 1771-78 CrossRef Medline

17. Hellström $P$, Klinge $P$, Tans J, et al. A new scale for assessment of severity and outcome in iNPH. Acta Neurol Scand 2012;126:229-37 CrossRef Medline

18. Warntjes JB, Leinhard OD, West J, et al. Rapid magnetic resonance quantification on the brain: optimization for clinical usage. Magn Reson Med 2008;60:320-29 CrossRef Medline

19. Alsop DC, Detre JA. Reduced transit-time sensitivity in noninvasive magnetic resonance imaging of human cerebral blood flow. J Cereb Blood Flow Metab 1996;16:1236-49 CrossRef Medline

20. Evans W. An encephalographic ratio for estimating ventricular enlargement and cerebral atrophy. Arch Neurol Psychiatry1942;47: 931-37 CrossRef

21. Virhammar J, Laurell K, Cesarini KG, et al. The callosal angle

AJNR Am J Neuroradiol 38:2081-88 Nov 2017 www.ajnr.org 
measured on MRI as a predictor of outcome in idiopathic normalpressure hydrocephalus. J Neurosurg 2014;120:178-84 CrossRef Medline

22. Fazekas F, Chawluk JB, Alavi A, et al. MR signal abnormalities at $\mathbf{1 . 5}$ $T$ in Alzheimer's dementia and normal aging. AJR Am J Roentgenol 1987;149:351-56 CrossRef Medline

23. Lundin F. Idiopathic Normal Pressure Hydrocephalus, Aspects on Pathophysiology, Clinical Characteristics and Evaluation Methods [PhD thesis]. Linköping: Linköping University; 2012

24. Hellstrom P. The Neuropsychology of Idiopathic Normal Pressure Hydrocephalus [PhD thesis]. Gothenburg: University of Gothenburg; 2011

25. Tullberg M, Hellström P, Piechnik SK, et al. Impaired wakefulness is associated with reduced anterior cingulate CBF in patients with normal pressure hydrocephalus. Acta Neurol Scand 2004;110:322-30 CrossRef Medline

26. Garrard P, Bradshaw D, Jäger HR, et al. Cognitive dysfunction after isolated brain stem insult: an underdiagnosed cause of long term morbidity. J Neurol Neurosurg Psychiatry 2002;73:191-94 CrossRef Medline

27. Ogino A, Kazui H, Miyoshi N, et al. Cognitive impairment in patients with idiopathic normal pressure hydrocephalus. Dement Geriatr Cogn Disord 2006;21:113-19 CrossRef Medline

28. E KH, Chen SH, Ho MH, et al. A meta-analysis of cerebellar contributions to higher cognition from PET and fMRI studies. Hum Brain Mapp 2014;35:593-615 CrossRef Medline

29. Moore DM, D'Mello AM, McGrath LM, et al. The developmental relationship between specific cognitive domains and grey matter in the cerebellum. Dev Cogn Neurosci 2017;24:1-11 CrossRef Medline

30. Malm J, Graff-Radford NR, Ishikawa M, et al. Influence of comorbidities in idiopathic normal pressure hydrocephalus: research and clinical care-a report of the ISHCSF task force on comorbidities in INPH. Fluids Barriers CNS 2013;10:22 CrossRef Medline

31. Krauss JK, Regel JP, Vach W, et al. White matter lesions in patients with idiopathic normal pressure hydrocephalus and in an agematched control group: a comparative study. Neurosurgery 1997;40: 491-95; discussion 495-96 Medline
32. Bastos-Leite AJ, Kuijer JP, Rombouts SA, et al. Cerebral blood flow by using pulsed arterial spin-labeling in elderly subjects with white matter hyperintensities. AJNR Am J Neuroradiol 2008;29:1296-301 CrossRef Medline

33. Xu G, Rowley HA, Wu G, et al. Reliability and precision of pseudocontinuous arterial spin labeling perfusion MRI on 3.0 T and comparison with 15O-water PET in elderly subjects at risk for Alzheimer's disease. NMR Biomed 2010;23:286-93 CrossRef Medline

34. Zaharchuk G, Bammer R, Straka M, et al. Arterial spin-label imaging in patients with normal bolus perfusion-weighted MR imaging findings: pilot identification of the borderzone sign. Radiology 2009;252:797-807 CrossRef Medline

35. Klinge PM, Berding G, Brinker T, et al. A positron emission tomography study of cerebrovascular reserve before and after shunt surgery in patients with idiopathic chronic hydrocephalus. J Neurosurg 1999;91:605-09 CrossRef Medline

36. Ivkovic M, Reiss-Zimmermann M, Katzen H, et al. MRI assessment of the effects of acetazolamide and external lumbar drainage in idiopathic normal pressure hydrocephalus. Fluids Barriers CNS 2015; 12:9 CrossRef Medline

37. Ambarki K, Wåhlin A, Zarrinkoob L, et al. Accuracy of parenchymal cerebral blood flow measurements using pseudocontinuous arterial spin-labeling in healthy volunteers. AJNR Am J Neuroradiol 2015;36:1816-21 CrossRef Medline

38. Wu WC, Lin SC, Wang DJ, et al. Measurement of cerebral white matter perfusion using pseudocontinuous arterial spin labeling $3 \mathrm{~T}$ magnetic resonance imaging: an experimental and theoretical investigation of feasibility. PLoS One 2013;8:e82679 CrossRef Medline

39. Skurdal MJ, Bjornerud A, van Osch MJ, et al. Voxel-wise perfusion assessment in cerebral white matter with PCASL at $3 \mathrm{~T}$ : is it possible and how long does it take? PLoS One 2015;10:e0135596 CrossRef Medline

40. Hellström P, Edsbagge M, Archer T, et al. The neuropsychology of patients with clinically diagnosed idiopathic normal pressure hydrocephalus. Neurosurgery 2007;61:1219-26; discussion 1227-28 CrossRef Medline 